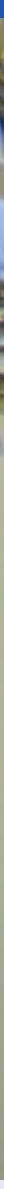

\title{
Protocollo della seduta straordinaria di due giorni della Camera medica svizzera
}

\author{
Giovedì e venerdì, 14 e 15 dicembre 2006, dalle 9.30 alle 17.00, \\ all'Unione postale universale (UPU), Berna
}

Annamaria Müller Imboden, segretaria generale FMH
Nota della redattrice del protocollo: contrariamente alle abitudini e per permettere una migliore comprensione delle consultazioni, in questo verbale i punti all'ordine del giorno sono riportati nell'ordine della discussione.

\section{Giovedi, 14 dicembre 2006}

\section{Benvenuto, comunicazioni, costituzione dell'ufficio}

(Regolamento di esecuzione della FMH, B II, art. 4.2)

Jacques de Haller, il presidente della FMH, apre la seduta alle 9.40, dà il benvenuto ai presenti e costata che il quorum è stato raggiunto e che quindi l'assemblea è regolarmente costituita. Seguono alcune osservazioni d'ordine tecnico e organizzativo, in particolar modo riguardo alla partecipa- zione di accompagnatori. In questo contesto ricorda il regolamento d'esecuzione in vigore e osserva che le persone che non hanno diritto di partecipazione devono essere in possesso di un invito da parte del Comitato centrale (CC).

J. de Haller informa che, rispetto alla convocazione, si devono aggiungere due complementi all'ordine del giorno, ovvero la presentazione dei nuovi collaboratori/delle nuove collaboratrici della Segreteria centrale (SG) al punto 1 dell'ordine del giorno, e la proposta della VEDAG al punto 3.3 dell'ordine del giorno. Su queste modifiche nessuno chiede la parola.

Annamaria Müller Imboden, la segretaria generale, presenta quindi i nuovi collaboratori della segreteria generale che hanno iniziato la loro attività dal giugno 2006. In ordine alfabetico sono: 


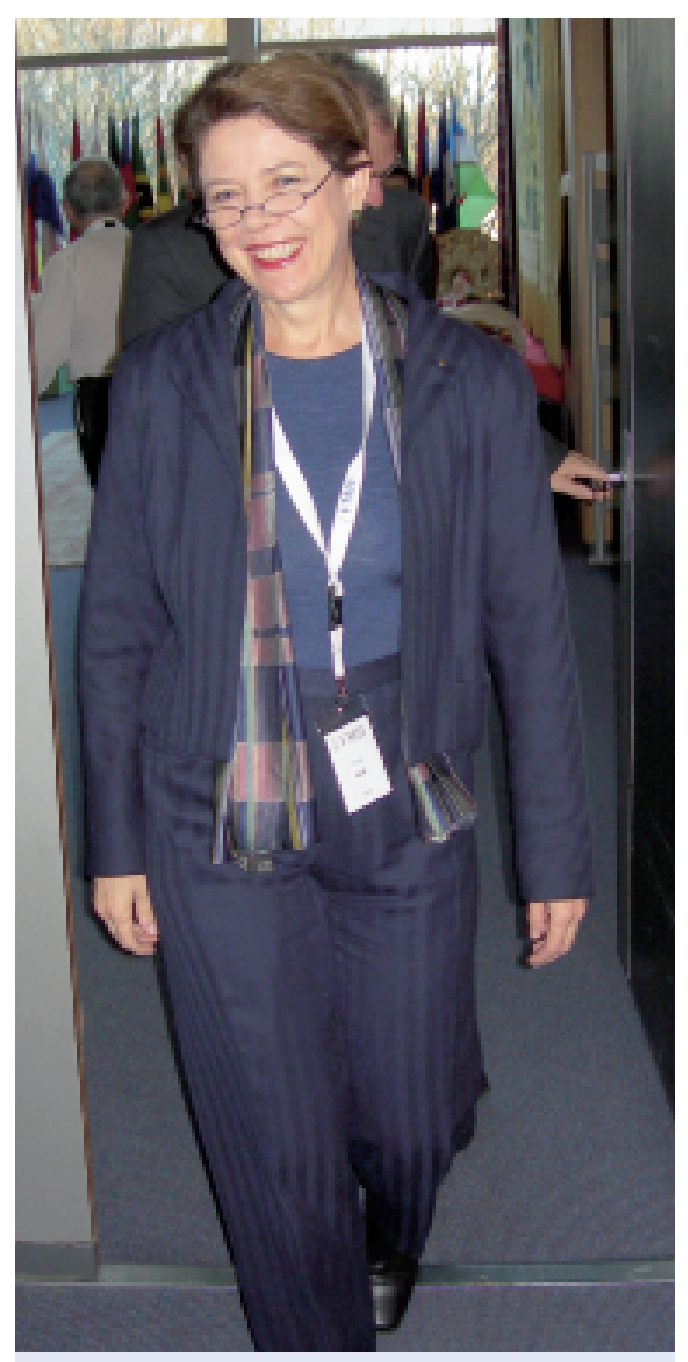

Brigitte Muff, appena eletta, ritorna alla Camera medica in qualità di nuovo membro del Comitato centrale della FMH.

- Gabrielle Heimgartner, segretaria al servizio giuridico;

- Esther Kraft, collaboratrice scientifica al dipartimento Dati, Demografia, Qualità (DDQ);

- Sandra Liechti, assistente in comunicazione;

- Franziska Müller, segretaria alla segreteria della direzione;

- Valérie Rothhardt, giurista al servizio giuridico;

- Esther Schmutz, segretaria alla segreteria della direzione;

- Barbara Schneider, collaboratrice specializzata al dipartimento della formazione, del perfezionamento professionale e dell'aggiornamento (FPA);

- Judith Wagner, responsabile del servizio eHealth;

- Olaf Wetter, collaboratore specializzato al servizio dell'amministrazione dei membri.
Sono designati come scrutatori: Luc Anex, Jean Biaggi, Roland Bingisser (sino a mezzogiorno)/ Brigitte Muff (a partire da mezzogiorno), Christoph Bossard, Franco Denti, François Héritier, Thomas Heuberger, Hansjakob Michel, Martin Rüegger.

\section{Elezioni}

\subsection{Sostituzione di un membro del Comitato centrale}

J. de Haller annuncia le dimissioni di Ludwig Theodor Heuss dopo dodici anni di attività in seno al Comitato centrale. Scusa la sua assenza e rende omaggio alla sua personalità critica e visionaria. Il commiato ufficiale si terrà in maggio. Dopo di che costata che a parte Brigitte Muff non è stata presentata nessun'altra candidatura per il seggio che si è liberato. Urban Laffer, presidente della $\mathrm{FMCH}$, presenta questa candidatura e schizza brevemente il curriculum di B. MUFF. Peter Studer, presidente dell'ASMAC, informa che l'ASMAC rinuncia a presentare un proprio candidato e che sostiene la candidatura di Brigitte Muff.

J. de Haller propone di votare per alzata di mano. Per l'approvazione di questa proposta è necessaria una maggioranza di due terzi. Nessuno chiede la parola. Senza voti contrari e senza astensioni la proposta è approvata. Dopo di che Brigitte Muff, che durante la votazione ha lasciato la sala, è eletta al Comitato centrale senza voti contrari e senza astensioni. I delegati la applaudono per la sua brillante elezione.

\subsection{Conferma dei delegati} per l'assemblea dei delegati FMH

È disponibile l'elenco dei delegati nominati dalle organizzazioni rappresentate all'assemblea dei delegati $(\mathrm{AD})$ e dei delegati supplenti. È stato completato con i nomi dei delegati supplenti ancora mancanti dell'OMCT e della MWS. Restano vacanti i seggi dei delegati supplenti dell'ASMAC e della SFSM. Nessuno chiede la parola a proposito dell'elenco. Nella votazione che segue l'elenco è approvato nella forma presentata senza che erano contati i voti e senza voti contrari e astensioni.

Per il periodo amministrativo dal 2007 al 2010 sono stati eletti delegati all'Assemblea dei delegati:

- ASMAC (5 seggi): Peter Studer, Winterthur; Patric Biaggi, Zurigo; Fabrice Dami, Carouge; Katharina Gasser, Berna; Oscar Matzinger, Losanna; delegato supplente: vacante;

- AMDHS (1 seggio): Carl Moll, Münsterlingen; delegato supplente: Hans-Ueli Würsten, Bienne; 
- VEDAG (5 seggi): Thomas Heuberger, Hilterfingen; Lukas Wagner, Birsfelden; Peter Wiedersheim, San Gallo; Urs Stoffel, Zurigo; Thomas Keller, Pfäffikon; delegato supplente: Beat Manser, Eich;

- SMSR (3 seggi): Charles-A. Favrod-Coune, Château-d'Oex; Jean-Daniel Schumacher, Tafers; Pierre-Alain Schneider, Veyrier; delegato supplente: Marc-Henri Gauchat, Sion;

- OMCT (1 seggio): Franco Denti, Barbengo; delegato supplente: Marco Ferrera, Melano;

- SFSM (5 seggi): Peter Buser, Basilea; Cem Gabay, Ginevra; Marcus Lütolf, Coira; Dominique Olgiati, Delémont; Jürg Nadig, Bülach; delegato supplente: vacante;

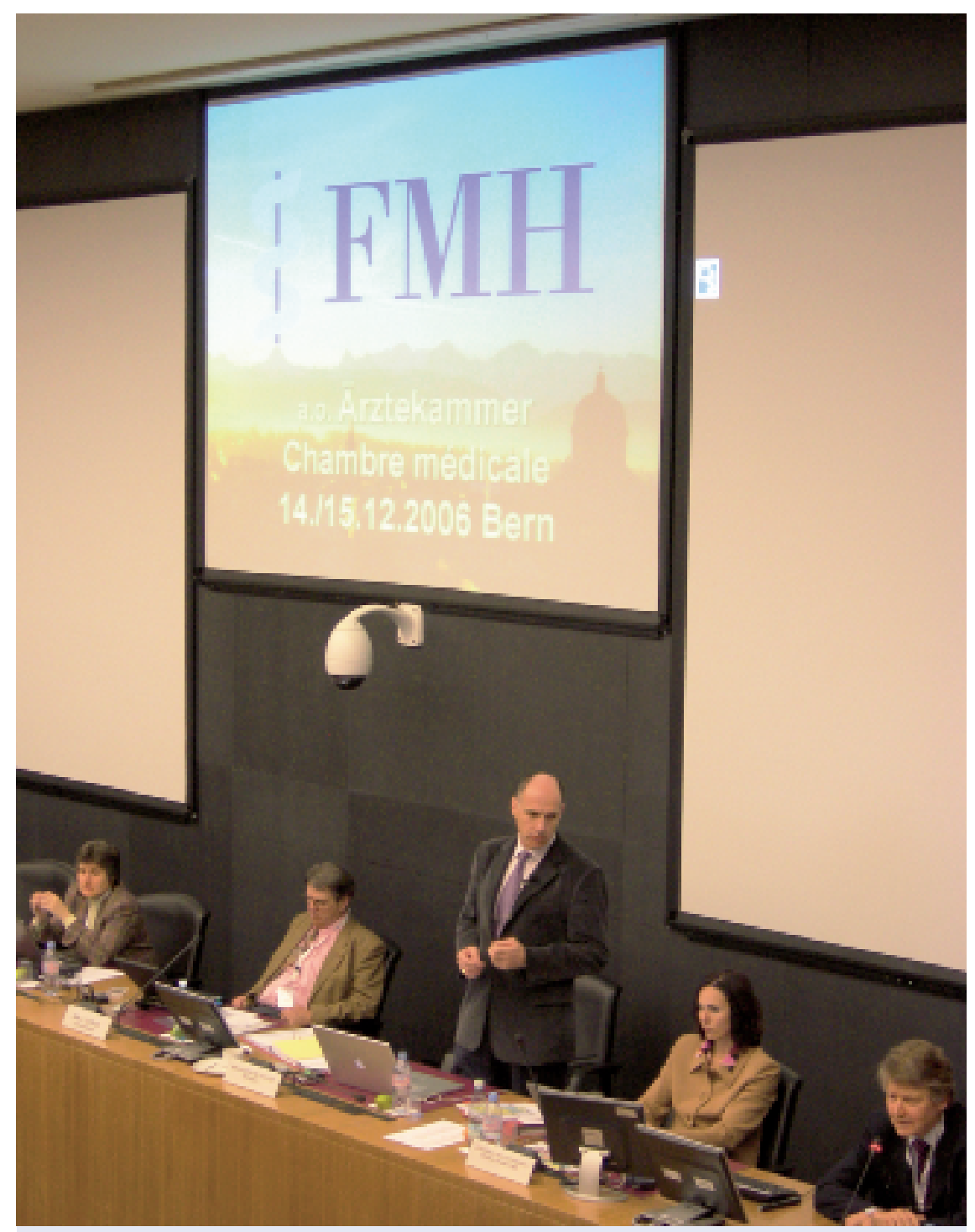

Susi Stöhr, Yves Guisan, Jacques de Haller, Annamaria Müller Imboden e Max Giger (da s. a d.) sul podio.
- FMPP (2 seggi): Hans Kurt, Soletta; Monique Gauthey, Ginevra; delegato supplente: Gianpiero Enderli, Locarno;

- FMCH (5 seggi): Urban Laffer, Bienne; Daniel Ackermann, Aarau; Jean Biaggi, Muri b. Bern; Michel Dutoit, Losanna; Guy-Peter Jenny, Heerbrugg; delegato supplente: Mario Litschgi, Sciaffusa;

- CMB (5 seggi): Franziska Zogg, Zugo; Marc Müller, Grindelwald; Werner Bauer, Küsnacht; Ueli Bürgi, Berna; Stephan Rupp, Einsiedeln; delegata supplente: Brigitte Saner, Olten;

- MWS (1 seggio): Marianna Bodenmann, Wetzikon; delegata supplente: Marianne Laifer, Basilea.

J. de Haller informa che la prima seduta dell'AD si terrà il 17 gennaio 2007 su invito del gruppo di coordinamento Riforma delle strutture.

\subsection{Elezione dei membri della Commissione di gestione}

La Commissione di gestione (CdG) sostituirà l'attuale Commissione delle finanze (COFI). Per la sua composizione sono state presentate cinque candidature, quanti sono i seggi da attribuire. Tra i candidati figurano due ex della COFI, ovvero Roland Schwarz e Adrian Sury. La candidatura del terzo ex membro della COFI, Charles-A. Favrod-Coune, ha dovuto essere ritirata all'ultimo momento a favore di quella di Lennart Magnusson, perché C.-A. Favrod-Coune - in quanto delegato $\mathrm{AD}$ - non può essere contemporaneamente membro della CdG. Gli altri due candidati, Thomas Kehl e Daniel Bielinski, sono stati sinora membri del gruppo di lavoro «Stabilizzazione del budget» e conoscono a fondo la situazione finanziaria della FMH. Nessuno chiede la parola sulle candidature e la proposta di J. de Haller di eleggere in blocco per alzata di mano, è approvata senza voti contrari o astensioni. Nella votazione che segue, sono eletti tutti e cinque i candidati senza voti contrari e con due astensioni. Per il periodo amministrativo dal 2007 al 2010

la CdG sarà composta da:

- Daniel Bielinksi, Oberdorf SO;

- Thomas Kehl, Davos Clavadel;

- Lennart Magnusson, Epalinges;

- Roland Schwarz, Muttenz;

- Adrian Sury, Locarno.

\subsection{Elezione di un nuovo vicepresidente} del Consiglio svizzero di deontologia

In un breve necrologio Max Giger, membro del CC e presidente della CPA, ricorda suo cognato, Dominique Haefeli, recentemente deceduto. La 


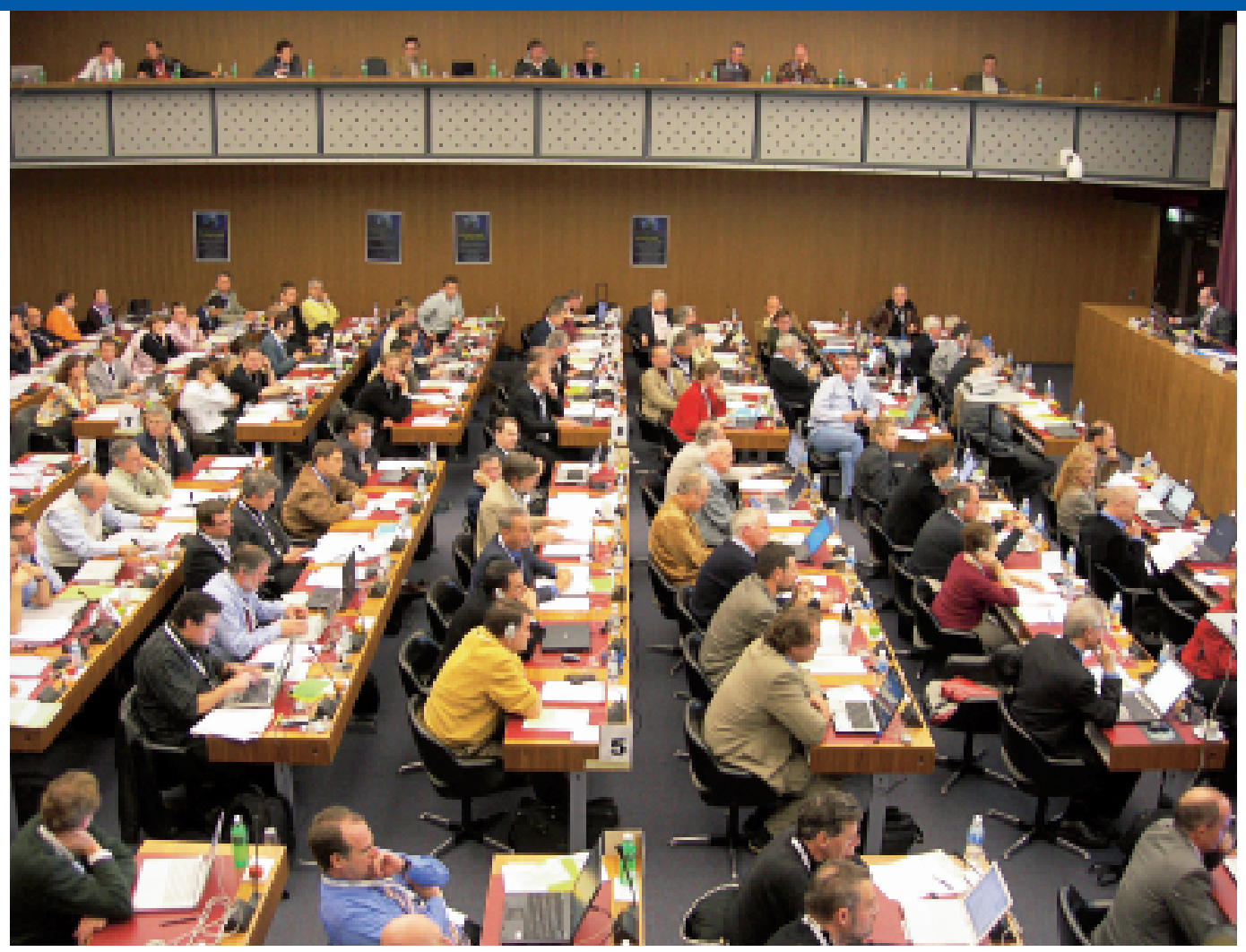

sua improvvisa scomparsa è il motivo per l'elezione suppletiva al Consiglio svizzero di deontologia. Per il seggio libero la SMSR propone ora Pierre-André Fauchère di Sion. Anche in questo caso ci si accorda - senza voti contrari e astensioni - per un'elezione per alzata di mano e P.-A. Fauchère è eletto con due voti contrari.

\section{Statuti FMH}

\subsection{Ammissione di «med-swiss.net» come organizzazione con diritto di partecipare alle consultazioni}

\section{La proposta n. 3.1/1 (Comitato centrale)}

mira a far aderire alla Camera medica svizzera, conformemente all'art. 25, cpv. 2 degli statuti, la Federazione delle Reti mediche svizzere «medswiss.net» come organizzazione con diritto di partecipare alle consultazioni. Nello stesso senso va la proposta n. 3.1/2 (P. Dreiding, Zurigo; A. von Weymarn, Turgovia), per la quale in futuro le Reti mediche svizzere assumeranno un ruolo importante e med-swiss.net dispone di una lunga esperienza in questo campo, della quale la FMH non può che approfittare. A causa della loro somiglianza, le due proposte sono riunite e - nonostante il fatto che sia espresso il timore di un «gonfiamento» pregiudizievole della Camera medica - sono approvate con 123 voti favorevoli, 9 contrari e 28 astensioni.
3.2 Adattamento degli statuti, del Regolamento di esecuzione, del Codice deontologico e del Regolamento del CSD

Con la proposta n. 3.2/1 (Comitato centrale) si vuole dare un nuovo nome al «Consiglio Svizzero di deontologia» che sia analogo per tutte e tre le lingue nazionali: «Standeskommission der FMH / Commission de déontologie de la FMH / Commissione di deontologia della FMH» con relativo adattamento degli statuti, del Regolamento d'esecuzione, del Codice deontologico e del Regolamento del CSD. Come motivo della proposta è addotto il fatto che secondo la sensibilità linguistica attuale il termine «Ehrenrat» è piuttosto ambiguo. Non è chiesta la parola e la proposta è approvata senza voti contrari o astensioni, per cui la maggioranza dei due terzi richiesta è raggiunta.

\subsection{Valutazione dei nuovi statuti}

La proposta n. 3.3/1 (C. Ramstein, VEDAG) chiede che, in vista delle elezioni generali degli organi della FMH nel maggio 2008, l'AD venga incaricata di valutare, nell'ambito di un comitato e con l'intervento di esperti esterni i diversi modelli direttivi per la FMH orientati verso l'avvenire. Questi modelli dovranno tener conto delle necessità e degli sviluppi attuali all'interno dell'associazione nonché della situazione attuale 
della politica della sanità. I primi risultati dovranno essere presentati alla Camera medica ordinaria del 3 maggio 2007, mentre alla Camera medica straordinaria del 6 dicembre 2007 dovrà essere proposto un modello adatto. Il richiedente spiega che nell'ambito della riforma delle strutture non si è tenuto abbastanza conto del CC e che si debba ora rimediare alla cosa.

A questa proposta il CC presenta una controproposta: desidererebbe che sia il Comitato centrale stesso ad esaminare il suo funzionamento e presenti una proposta alla Camera medica del mese di maggio. J. de Haller spiega questo atteggiamento precisando che il modo di funzionare dell'AD non è stato ancora abbastanza provato e che non si dovrebbe disconoscere al CC la possibilità di riflettere su se stesso. Teme, inoltre, che riducendo il CC si blocchi la rielezione di nuovi membri. Nella discussione che segue risulta che una maggioranza degli intervenenti mostra comprensione per la controproposta del CC, ma che sostiene la proposta originale della VEDAG. Siccome il CC parteciperà alle sedute dell'AD, potrà intervenire senza problema. Il timore che le nuove strutture non ancora consolidate non siano all'altezza della situazione e un nuovo strapazzamento delle finanze della FMH a causa di nuovi incarichi ai consulenti, non impedisce ai delegati alla Camera di seguire la proposta della VEDAG con 89 voti favorevoli e 55 contrari.

\section{Codice deontologico della FMH}

\subsection{Adattamento alla revisione degli statuti (Commissione di deontologia dell'AMDHS)}

Secondo quanto affermato da Hanspeter Kuhn, vicesegretario generale, la proposta $n$. 4.1/1 (Comitato centrale) è di natura essenzialmente tecnica. A causa della modifica degli statuti dell'AMDHS, è necessario che in quanto organizzazione di base disponga di una commissione di deontologia. Il codice deontologico della FMH deve essere adattato all'art. 43 nel modo seguente:

«Il codice deontologico è vincolante per tutti i membri della FMH, salvo nel caso in cui il diritto sanitario cantonale preveda delle disposizione contrarie. Le società mediche cantonali, l'ASMAC rispettivamente l'AMDHS informano su eventuali divergenze esistenti.

Le società mediche cantonali, l'ASMAC e l'AMDHS si assicurano che il codice deontologico venga osservato dai loro membri. A questo scopo costituiscono un organo apposito (qui di seguito chiamato «commissione di deontologia $)$, incaricato di giudicare le infrazioni al codice deontologico. Contro le decisioni della commissione di deontologia si può ricorrere presentando un reclamo presso il Consiglio svizzero di deontologia.» (in questo senso si devono completare gli art. 44 e 47 segg. del codice deontologico).
Su quest'argomento nessuno chiede la parola. La proposta è approvata senza voti contrari e con due astensioni.

\section{Rimborso del prestito all'EMH}

\section{Con la proposta $n$. 5/1 (Comitato centrale)}

si chiede alla Camera medica l'approvazione del rimborso, da parte della casa editrice EMH SA, del prestito alla EMH SA ripreso da FMH Services il 2 dicembre 2005. Il rimborso sarà effettuato al prezzo di Fr. 903 804.- (valore rettificato corrispondente all'80\% del valore nominale di Fr. 1129 755.-). J. de Haller, che è anche membro del consiglio d'amministrazione della EMH, presenta la proposta e coglie l'occasione per spiegare in questo contesto ancora una volta i «lavori di aggiornamento» delle diverse partecipazioni della FMH, che sono stati l'oggetto della Camera medica straordinaria del 1 e del 2 dicembre 2005. Il prestito sopraccitato rappresenta l'ultimo elemento rimasto del confuso costrutto finanziario. J. de Haller aggiunge, che il rimborso proposto non causerà nessuna perdita alla FMH, perché a suo tempo il rimborso era già stato ripreso al valore rettificato. Per l'EMH - che fortunatamente è riuscita a disporre dei relativi liquidi - è invece determinante non dover pagare il totale del valore nominale, perché in questo modo il risanamento finanziario dell'impresa sarebbe stato minacciato. Anche Roland Schwarz, presidente della COFI e membro della «commissione di fiducia» di allora, raccomanda di approvare la proposta. Werner Bauer, che aveva presieduto la commissione, sostiene questa proposta. Ma prima di passare ai voti, sono poste delle domande sull'impegno della FMH presso FMH Services. J. de Haller, assistito da A. Müller Imboden, spiega che si tratta esclusivamente di un sostegno morale, che implica principalmente l'utilizzazione del logo «FMH». In questo senso è stata stipulata una convenzione ad hoc. Non c'è nessuna implicazione finanziaria. La politica di partecipazione della $\mathrm{FMH}$, inoltre, è regolarmente oggetto delle consultazioni del CC, che mira a limitare i mandati doppi, o addirittura a eliminarli. Si è anche discusso dell'impegno della FMH alla EMH. I romandi fanno notare che la FMH dovrebbe intervenire anche a favore di altre case editrici - in particolar modo per Medecine \& Hygiène. J. de Haller spiega i legami particolari con l'EMH come joint venture con Schwabe e l'esclusività di questo legame per via della pubblicazione degli organi ufficiali dei medici svizzeri: «Bollettino dei medici svizzeri», «Forum medicale svizzero»e «Swiss Medical Weekly». La proposta è quindi approvata con 2 voti contrari e 5 astensioni. 
Segue una mozione d'ordine (O. Matzinger, ASMAC), secondo la quale si deve votare sul consolidamento del budget prima di decidere su altri progetti. Questa proposta è tuttavia respinta dalla maggioranza dei delegati, senza che siano contati i voti.

\section{Health Professional Card (HPC) - Carta di professionista della sanità (CPS)}

Per incominciare, J. de Haller indica che il progetto HPC - su cui una decisione di massima era stata presa dalla Camera medica straordinaria del dicembre 2005 e per il quale era stato dato l'incarico di elaborare un primo concetto generale rappresenta un'occasione eccezionale per la FMH per trasformare la sua carta di membro in una carta di medico. La finestra di tempo non rimane però aperta a lungo, perché l'industria è impaziente di impegnarsi in questo affare. Judith Wagner, la responsabile del settore eHealth, presenta quindi il progetto secondo il suo concetto generale che era stato consegnato ai delegati della Camera medica. Gli elementi centrali ne sono l'elaborazione della carta in diverse fasi di sviluppo (fase iniziale, fase 1 e fase 2), la realizzazione secondo un piano a tappe e la realizzazione di una carta a struttura aperta, che dovrebbe permettere alla FMH di rimanere indipendente nei confronti di produttori e tecnologia. A. Müller Imboden presenta le varianti di finanziamento proposte (con o senza ricorso ad un contributo dagli accantonamenti PR).

In proposito, sono state fatte in seguito numerose domande. La FMH è legittimata a emettere una carta di medico svizzero (anche per i non membri)? Quanto vincolante sarà la carta e come verrà regolato il suo riconoscimento sul piano giuridico? È necessaria l'HPC oppure si tratta soltanto di un gadget marketing costoso? Alla fine non sarà la Confederazione, in quanto «custode del Gral delle professioni mediche», a decidere chi deve emettere l'HPC, contrastando così qualsiasi dichiarazione della FMH? L'emissione di un'HPC da parte della FMH non vuol dire ammettere l'introduzione della carta di assicurato o addirittura una carta salute? L'HPC deve essere così cara e quali sono i costi conseguenti da essa indotti? Con chi collaborerà la FMH? Secondo l'argomento della domanda rispondono J. de Haller, O. Kappeler, A. Müller Imboden oppure J. Wagner: la Confederazione ha segnalato che non soltanto accetterebbe, ma che sarebbe addirittura lieta, se la FMH emettesse e gestisse l'HPC per il corpo medico. Il carattere vincolante della carta dipende da quanto vincolante è la sua utilizzazione e dalle premesse giuridiche. Se la FMH non coglie adesso l'occasione che le si offre - a favore della quale un anno fa la Camera medica si era espressa in modo molto chiaro - perderebbe una possibilità unica di emettere questa carta, che rappresenta molto più di un semplice gadget di marketing. I costi indicati si basano su delle offerte indicative che sono

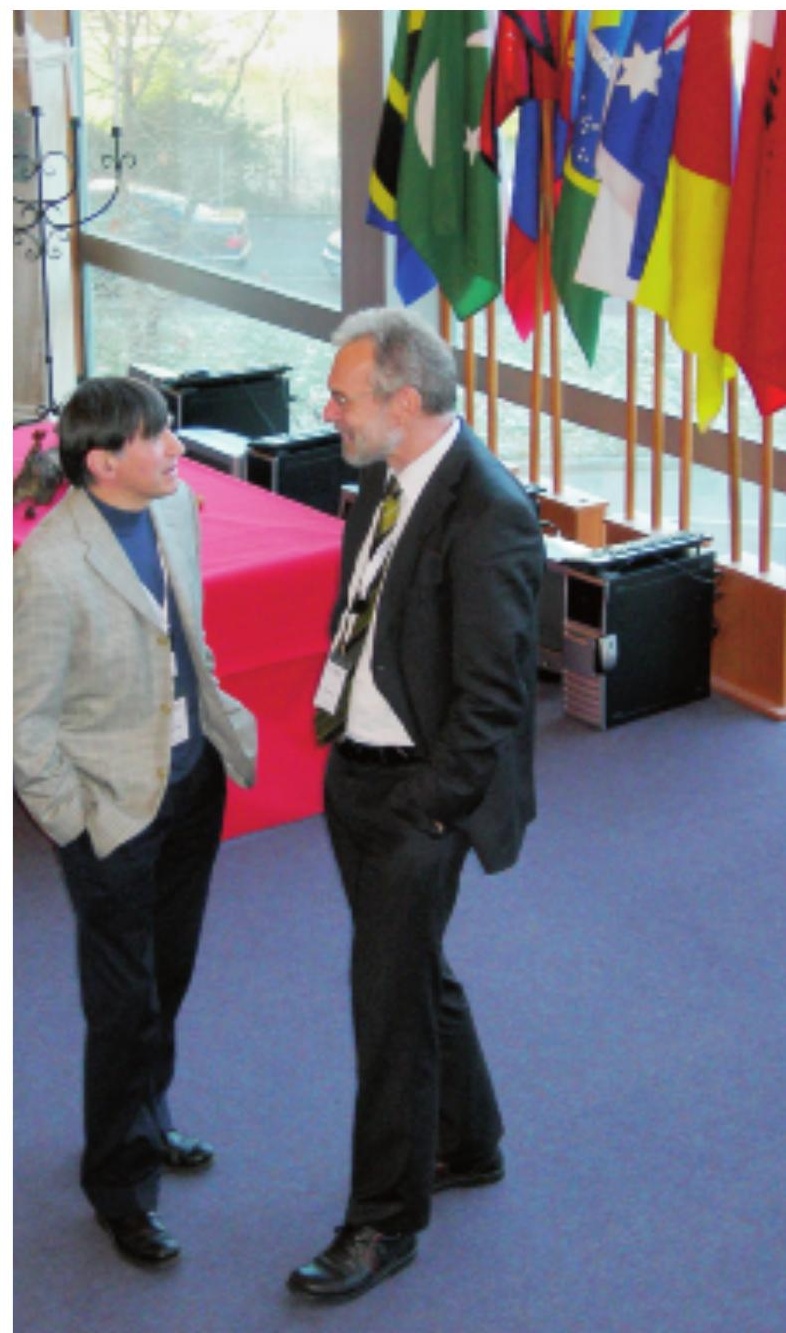

Olivier Kappeler, membro del Comitato centrale (a destra), in piena discussione con Roland Schwarz, presidente della CoFi.

state chieste, ma un bando pubblico non c'è ancora stato, in quanto è necessario che la Camera medica dia prima il suo assenso. La gestione della carta avverrà nell'ambito dell'amministrazione dei membri e non causerà nessun costo supplementare. I costi conseguenti si limiteranno quindi alla sostituzione della carta. L'ASMAC afferma di non sostenere il progetto, perché considera che il finanziamento non sia chiaro e non si sia trattato, come aveva richiesto, prima del consolidamento del budget (che mira ad una riduzione del rimborso delle quote dei membri). 
Prima di passare alle singole proposte J. de Haller mette ai voti il concetto generale, che è approvato con 8 astensioni e nessun voto contrario. Si passa quindi alla votazione sulle varianti di finanziamento. Con 73 contro 69 è approvata l'inclusione dell'utilizzazione di una buona parte delle riserve accantonate PR (variante B).

Si passa quindi a trattare la proposta $n$. 6/2 (J. Schlup, Berna) con la quale si vuole aggiungere alle proposte del Comitato centrale un quarto punto, perché la questione è complessa e altamente imponderabile. L'AD dovrebbe seguire l'evoluzione della situazione ed essere eventualmente in grado di reagire nell'ambito delle sue competenze previste dagli statuti. La proposta è approvata dal Comitato centrale senza discussione alcuna, perché in tutti i casi era già previsto cosi.

\section{La proposta n. 6/1 (Comitato centrale)}

viene modificata in questo senso e messa in votazione nella forma seguente:

1. approvazione dell'attuale concetto generale per il progetto per una «Health Professional Card - carta elettronica di medico della FMH»;

2. approvazione del credito relativo a questo progetto con la variante di finanziamento B (credito speciale per tre anni di Fr. 25.- per le categorie di contributo 1 e 2; Fr. 12.- per le categorie 3 e 4, nonché Fr. 6.- per le categorie 5 e 6 ; un contributo una tantum di Fr. 1000000.- prelevato dagli accantonamenti per $\mathrm{PR}$ );

3. incarico al Comitato centrale di realizzare il progetto e

4. informare regolarmente l'AD sui progressi del progetto e sulle spese già effettuate nell'ambito del credito per il progetto (stato dell'utilizzazione del credito).

La proposta è approvata con 103 voti favorevoli, 49 voti contrari e 9 astensioni.

\section{Informazioni dai vari settori di attività}

\subsection{Salute e prevenzione}

René Raggenbass, membro del Comitato centrale e responsabile del settore «Salute e prevenzione» e Barbara Weil, responsabile del dipartimento «Prevenzione» presentano i progetti in corso del loro settore e quelli previsti. Alla loro base c'è l'intenzione chiara del CC di promuovere una posizione più attiva della FMH. Sono presentati i temi seguenti:

- creazione di una commissione Prevenzione (in quanto commissione consultiva del CC e del settore di attività);
- legge sulla prevenzione: calendario e intervento;

- gruppo di lavoro Protezione della maternità: obiettivi, calendario e composizione;

- malattie trasmissibili: campagne dell'UFSP nel 2007 nel campo della prevenzione HIV;

- malattie croniche, non trasmissibili: «Peso corporeo sano» e CardioVascSuisse;

- politica in materia di droga: comunità nazionale di lavoro Politica in materia di droga;

- salute psichica: promozione della salute Svizzera e Ipsilon (Associazione Iniziativa per la prevenzione del suicidio in Svizzera).

I delegati ringraziano per la relazione con un applauso.

\subsection{Formazione, perfezionamento professionale e aggiornamento}

Max Giger fa un rapporto sul settore «Medical Education». Per il 2006 presenta il settore dal punto di vista quantitativo (per esempio per quel che concerne titoli, programmi, centri di perfezionamento) e alcuni dati strutturali del CostCenter FPA (prestazioni e risorse). Ricorda quindi i progetti trattati

- programma eLearning (formazione on line) per la radioprotezione in collaborazione con l'UFSP;

- risultati dell'inchiesta tra gli assistenti sulla qualità del perfezionamento professionale;

- progetti di valutazione in collaborazione con l'Istituto per la formazione medica dell'Università di Berna (IML).

Per il 2007 prevede dei progetti nei campi seguenti:

- valutazione;

- economia della sanità e etica;

- ottimizzazione della comunicazione con i responsabili dei centri di perfezionamento professionale;

- revisione in corso dei programmi di perfezionamento professionale;

- inchiesta tra gli assistenti sul perfezionamento professionale;

- visite in occasione dei cambiamenti di responsabile dei centri di formazione;

- promozione delle reti di perfezionamento professionale;

- inchiesta, 12-18 mesi dopo l'esame di specializzazione, relativa al programma di perfezionamento professionale;

- sostegno da parte delle società mediche specialistiche in materia di aggiornamento. 
Secondo M. Giger i challenge per la formazione professionale medica nel 2010 saranno:

- l'incremento dell'efficienza del perfezionamento professionale, in particolare in relazione all'introduzione del DRG (sistema di retribuzione mediante forfait) negli ospedali;

- l'accreditamento dei 43 programmi di perfezionamento professionale conformemente alla LPMed e

- autodeterminazione dell'aggiornamento.

Anche questa relazione è applaudita.

Nella discussione che segue sono toccati diversi argomenti complessi:

- garanzia della formazione e del perfezionamento professionale del medico. I rappresentanti cantonali sono invitati a mettersi in contatto con le autorità in modo che il perfezionamento professionale sia integrato negli incarichi di prestazione affidati agli ospedali (allo scopo di assicurarne il finanziamento!);

- esami di specializzazione: differenze di qualità tra la Svizzera e l'UE; importazione di medici dall'estero e le implicazioni che ne risultano;

- integrazione degli assicuratori e chiaro riconoscimento della formazione professionale dei medici.

\subsection{Tariffe e contratti}

Franco Muggli, membro del Comitato centrale e responsabile del settore «Tariffe e contratti» prende la parola e inizia con il ringraziare i suoi colleghi del CC, il responsabile e i collaboratori del servizio Tariffe e il presidente della FMH per la loro collaborazione costruttiva, anche se a volte critica. Informa, quindi, sulle attività seguenti:

Organizzazione del settore Tariffe \& Contratti Creazione dell'«Ufficio delle tariffe» con gli obiettivi seguenti:

- migliorare la visione d'insieme e la determinazione delle priorità;

- migliorare la coordinazione facendo intervenire in modo continuo le federazioni, la Conferenza delle società mediche cantonali (CSMC) e il settore DRG;

- raggruppare sul piano organizzativo le zone contrattuali rilevanti per le tariffe;

- assicurare la continuità nella comunicazione;

- suddividere i settori e le competenze.

Convenzione prestazioni-prezzo nel settore AINF/AM/AI con i punti forti seguenti:

- parità dei dati;

- costi annuali di trattamento come indice di riferimento;
- settori di osservazione: ambulatoriale e degenza;

- limiti d'intervento differenziati;

- modello di prognosi.

\section{Reengineering III}

Contrarietà, imponderabilità e criteri per soluzioni.

\section{Banca dati valori intrinseci}

(questa parte viene presentata da Olivier Kappeler, membro del Comitato centrale e della task force Tariffe):

- dall'ottobre 2004 è disponibile la banca dei dati sul valore intrinseco secondo le disposizioni pattuite contrattualmente;

- le convalide esterne sono già state effettuate con successo;

- né santésuisse né la CTM utilizzeranno tuttavia ai primi del 2007 la banca dei dati sul valore intrinseco per la convalida delle fatture;

- continua quindi a non essere possibile rinviare le fatture;

- le posizioni di diritti acquisiti devono essere, tuttavia, aggiornate.

I delegati ringraziano O. Kappeler per la relazione con un applauso. Un delegato esprime il suo disappunto per la coordinazione insufficiente tra le diverse zone contrattuali.

\subsection{Dati, demografia e qualità}

Martina Hersperger, responsabile del dipartimento Dati, demografia e qualità informa sulle attività nel suo settore.

Gruppo di lavoro qualità (GLQ)

- commissione consultiva del Comitato centrale;

- redazione dei documenti per una presa di posizione;

- elaborazione di griglie di criteri per la valutazione di progetti relativi alla qualità;

- panoramica sul lavoro di qualità nazionale e internazionale e posizionamento della FMH.

\section{Progetto ReMed}

- elaborazione di uno studio sulla fattibilità;

- effettuazione di un workshop;

- concetto per progetto pilota.

\section{Statistica dei medici}

- ripresa dal settore DDQ della statistica medica tenuta sino ad ora da Jürg Jau;

- progetto Revisione statistica medici (in vigore a partire dal 2008). 
Sistemi indicatori di morbilità

- panoramica bibliografica dell'Istituto di medicina sociale e preventiva dell'Università di Berna (ISPM);

- pubblicazione dei risultati nel gennaio 2007 nel Bollettino dei medici svizzeri.

Partecipazione del settore DDQ a progetti esterni

- modulo «medicina di base» nell'ambito del progetto di ricerca finanziato dal Fondo nazionale svizzero, relativo ai criteri che determinano l'evoluzione della carriera dei giovani medici;

- studio relativo alla gestione dei rischi negli ospedali svizzeri: situazione attuale e punti di vista soggettivi sull'esempio del sistema di dichiarazione degli incidenti tecnici;

- progetto concernente la demografia medica: offerta e domanda di prestazioni ambulatoriali in Svizzera.

Olivier Kappeler, che riprende il settore DDQ dal membro dimissionario del CC Ludwig Theodor Heuss, ringrazia il suo predecessore - nonostante sia assente - per i lavori preparatori portati avanti

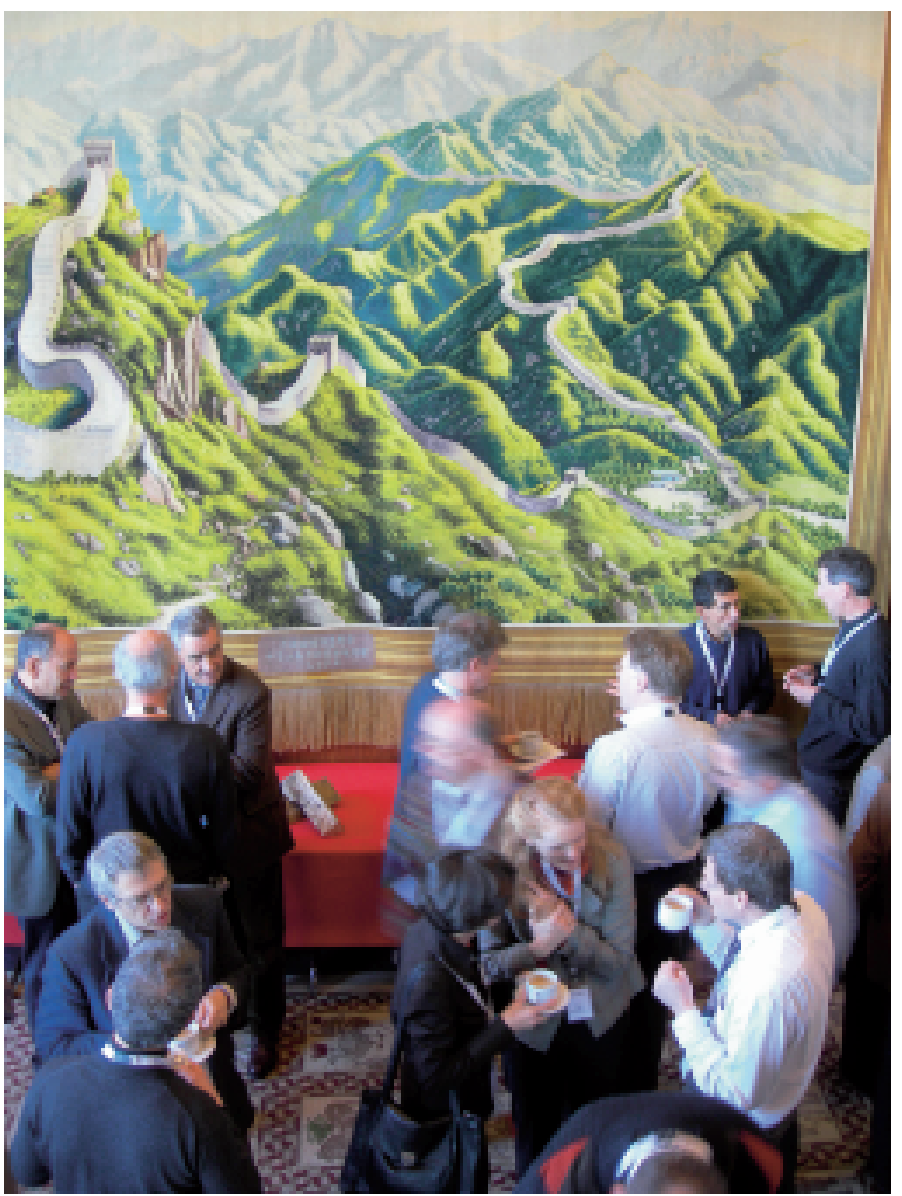

con molto impegno. Un ringraziamento nello stesso senso va anche all'attenzione della responsabile del dipartimento.

Siccome incomincia a farsi tardi, J. de Haller propone di continuare la seduta con i punti politici all'ordine del giorno, in modo che nella giornata seguente della Camera medica rimanga tempo a sufficienza per discutere il budget. Nessuno si oppone alla proposta.

\subsection{Cassa unitaria}

J. de Haller informa sul testo presentato alla Camera medica per approvazione, relativo alla presa di posizione sull'iniziativa inerente alla cassa malattia unitaria, che ha elaborato un gruppo di lavoro composto in modo rappresentativo. Ci sono diverse proposte che vengono presentate in breve qui di seguito:

\section{La proposta n. 9.2/1 (Comitato centrale)}

auspica - sensatamente - l'approvazione del testo presentato ai delegati. Non da luogo a ulteriori spiegazioni.

\section{La proposta $n$. 9.2/2}

(C.-A. Favrod-Coune, Vaud)

è presentata sotto forma di una lettera e chiede la libertà di voto per la votazione dell'11 marzo 2007. Come motivo adduce il fatto che i medici vodesi, nell'ambito di un'indagine consultiva, si sono espressi a favore dell'iniziativa, perché vedono in essa una bella lezione all'indirizzo degli assicuratori e del potere politico. Se la Camera medica dovesse consigliare di votare no all'iniziativa, i suoi avversari potrebbero vedervi il segno di una scissione del corpo medico. È quindi importante che ogni società cantonale possa esprimere la sensibilità dei suoi membri senza pressione. A questo scopo la presa di posizione della FMH deve limitarsi alla parte analitica del documento, che d'altronde è molto lodata.

\section{La proposta n. 9.2/3 (A. Souche, Ginevra)} va nella stessa direzione. Anch'egli propone la libertà di voto e invita a modificare la raccomandazione a pagina 2 , in modo che sia messa in evidenza l'importanza dell'iniziativa. Il motivo della sua proposta sta anche nella posizione in linea di massima favorevole della società medicha cantonale rispetto all'iniziativa. I medici ginevrini auspicano anche che la FMH, prima di prendere posizione, organizzi un'inchiesta fra tutte le società mediche cantonali per conoscere il loro parere e ne riassuma le risposte ottenute. 
La proposta n. 9.2/4 (J. Nadig, Oncologia) chiede che la FMH rinunci del tutto ad una raccomandazione di voto. La FMH dovrebbe, invece, spiegare in una presa di posizione pubblica i vantaggi e gli svantaggi dei due sistemi e mostrare la responsabilità del medico per quel che concerne una distribuzione equa dei mezzi finanziari nel settore della sanità. Alla base della proposta c'è il timore che le casse malattie obblighino indirettamente il corpo medico a razionare le cure, mentre la cassa unitaria non può mantenere le promesse fatte. Il corpo medico non dovrebbe occuparsi delle questioni relative al finanziamento, ma adoperarsi per un trattamento adeguato e un accesso con diritti uguali per tutti alle prestazioni mediche e assicurarsi che non sia strumentalizzato.

Nel dibattito che segue ci si complimenta per l'analisi e la valutazione della situazione, ma i delegati non concordano sulle conclusioni. L'ASMAC, come i vodesi e i ginevrini, propone la libertà di voto. L'ASMAC non ci tiene a provocare una discussione del tipo «Röstigraben». Altri intervenenti chiedono, invece, un posizionamento chiaro da parte della FMH, come ci si deve attendere da parte di un partner nella sanità pubblica da prendere sul serio. L'argomento principale degli oppositori all'iniziativa è il timore di una medicina di stato come anche l'obiezione che non si può dire sì ad un cattivo testo di iniziativa, soltanto perché il sistema attuale non ci conviene. E chi non vota o vota scheda bianca, lascia il campo libero alle minoranze che esprimono un'opinione chiara. Ma che cosa bisogna votare? Un no nelle urne presenta lo svantaggio che l'opinione pubblica lo potrebbe considerare un sì al sistema attuale. Si discute anche sull'astensione di voto rispetto alla libertà di voto. Mentre la libertà di voto lascia la scelta a ciascuno di decidere, la raccomandazione di astenersi dal votare esprime chiaramente una protesta. Sul piano politico è un segnale più forte. J. de Haller mette a fuoco tre opinioni: una per il no, una per l'astensione e una per la libertà di voto. Un delegato descrive la discussione come una «scelta tra il colera e la peste».

Una proposta ad hoc (P. de Vevey, Vaud) chiede l'effettuazione di una votazione generale. La proposta è respinta con 133 voti contrari, 16 voti favorevoli e 2 astensioni. [Osservazione della redattrice del protocollo: in caso di approvazione questa proposta avrebbe dovuto essere dichiarata non applicabile per dei motivi di procedura, perché il termine previsto dagli statuti per una votazione generale avrebbe oltrepassato la data della votazione popolare.]
J. de Haller presenta alla fine una soluzione alternativa per l'ultima frase della raccomandazione: «La Camera medica raccomanda quindi l'astensione alla votazione.» I delegati votano per sapere se il termine «astensione» debba essere sostituito da «libertà di voto». Quest'alternativa è approvata con 78 voti favorevoli, 77 contrari e 3 astensioni. C.-A. Favrod-Coune e A. Souche, che avevano presentato rispettivamente la proposta n. 9.2/2 e la 9.2/3, ritirano la loro proposta dichiarandosi soddisfatti. Anche J. Nadig (proposta $\mathrm{n}$. 9.2/4) si dichiara soddisfatto. La proposta del CC (proposta n. 9.2/1) è approvata nella sua forma modificata. Ma siccome non è sicuro - come lo costata una mozione d'ordine (T. Keller, Svitto) - che la volontà della Camera medica si sia espressa correttamente - ai delegati è proposto di ripetere la votazione precedente. Questi si oppongono a procedere in questo modo con 90 voti contrari e 54 favorevoli.

Sono le 17.00 e J. de Haller chiude la seduta indicando che il giorno dopo i punti all'ordine del giorno rimanenti saranno trattati nell'ordine indicato.

\section{Venerdi, 15 dicembre 2006}

\section{Benvenuto, comunicazioni, costituzione dell'ufficio}

(Regolamento di esecuzione della FMH, B II, art. 4.2)

Jacques de Haller apre la seduta alle 9.40 e costata che il quorum è stato raggiunto e che quindi l'assemblea è regolarmente costituita. Chiede, inoltre, ai presenti di rimanere sino alla fine della seduta, perché ha da fare una comunicazione importante.

Vengono designati come scrutatori: Christoph Bosshard, Blaise Bourrit, Marc Egli, Martin Gubler, François Héritier, Thomas Heuberger, Hans Kurt, Martin Rüegger, Peter Tschudi.

\section{Consolidamento del budget}

J. de Haller ricorda gli incarichi dati dalla Camera medica del maggio 2006 e presenta i risultati dei due gruppi di lavoro «Rimborso» e «Stabilizzazione».

Roland Schwarz presenta la proposta del gruppo di lavoro «Rimborso» da lui presieduta. Questa proposta mira ad una riduzione della metà degli sconti a lungo termine e segue il principio secondo il quale ai membri FMH che incominciano il loro perfezionamento professionale al 1 gennaio 2007 o dopo questa data, sarà rimborsato il loro contributo membro (Fr. 1000.-) soltanto al momento dell'acquisizione del titolo 
di specialista, ma non più in seguito. I membri FMH che hanno incominciato il loro perfezionamento professionale prima del $1^{\circ}$ gennaio 2007 continueranno a beneficiare del sistema valido sinora per al massimo 7 anni (diritti acquisiti). Al fine di ridurre di metà il deficit per gli anni dal 2007 al 2017, viene proposto un aumento immediato delle quote di membro di Fr. 25.- all'anno (calcolato proporzionalmente per le diverse categorie di quote). Questa proposta di soluzione del gruppo di lavoro «Rimborso» si basa sull'idea che da un lato convenga continuare ad assumersi la responsabilità del perfezionamento professionale e che, dall'altro, il settore del perfezionamento professionale può contribuire anch'esso alla riduzione del deficit. Si devono, inoltre, rispettare le leggi in vigore, le decisioni statutarie e le promesse fatte. R. Schwarz segnala tra l'altro che rinunciando interamente al rimborso, sarebbe possibile ridurre il deficit strutturale del budget senza dover prendere altri provvedimenti.

Daniel Bielinski, portavoce del secondo gruppo di lavoro «Stabilizzazione», presenta i risultati del gruppo. Dopo aver ricordato qual era il mandato, presenta come è composto il gruppo e il suo modo di procedere. Spiega, infine, la proposta del gruppo che tiene conto delle conclusioni del gruppo di lavoro «Rimborso». In relazione all'analisi accompagnata da un esperto esterno, il gruppo di lavoro ha costatato che la gestione dei centri di costo e dei mezzi finanziari è effettuata con la massima serietà. Si complimenta in proposito con la SG. Secondo lui il pacchetto di misure proposte:

- è equilibrato;

- permette di colmare la lacuna finanziaria senza riduzione delle prestazioni;

- prevede soltanto un aumento moderato delle quote (quelle proposte dal gruppo di lavoro «Rimborso»);

- include diversi settori di attività;

- è presentato all'unanimità dal gruppo di lavoro «Stabilizzazione»;

- comprende, oltre alla diminuzione progressiva dei rimborsi, una riduzione dei compiti del CC, delle misure di ottimizzazione in seno alla SG e un ricorso alle riserve del settore FPA (rispettivamente a quelle del contributo di solidarietà che giuridicamente non sono contestate);

- è pianificato a tappe e permette di effettuare, nel lasso di tempo previsto, dei risparmi per un importo di 1,5 milioni di franchi all'anno;

- poiché le misure non possono essere divulgate nei dettagli, dovrà essere gestito «fiduciariamente» dal CC, mentre la Commissione di ge- stione si incaricherà di sorvegliarne la realizzazione.

I delegati prendono nota dei risultati dei due gruppi di lavoro e ringraziano i loro rappresentanti. La misura prevista dal gruppo di lavoro «Stabilizzazione», ovvero l'utilizzazione delle riserve FPA, solleva delle animate discussioni. I giovani medici dichiarano di opporsi con estrema risolutezza ad una riduzione dei rimborsi. Qualcuno pensa addirittura che questi rimborsi siano il solo motivo di aderire alla FMH. M. Giger, nella Sua funzione di presidente della CPA, chiede all'assemblea di osservare le decisioni della Camera medica del 2001 e metta in guardia contro uno smantellamento del perfezionamento professionale utilizzando le sue riserve. Numerosi intervenenti di fama sostengono questo punto di vista. J. de Haller dichiara, da parte sua, di non essere disposto a realizzare soltanto alcuni aspetti delle misure proposte nel pacchetto. Queste misure formano un insieme equo in termine di sacrifici e comprendono anche, per il perfezionamento professionale, delle soluzioni di compromesso accettabili. Secondo lui non si può parlare di rifiuto. Numerosi delegati alla Camera medica considerano, tuttavia, che il solo fatto di parlare di un eventuale trasferimento dei fondi della CPA rappresenta già un segnale politico che gli oppositori della FMH potrebbero interpretare a suo svantaggio. Inoltre, si critica il fatto che le misure del gruppo di lavoro «Stabilizzazione» siano state abbozzate solamente in modo rudimentale e che i delegati alla Camera medica non abbiano avuto la possibilità di farsi un'idea approfondita. Yves Guisan, vicepresidente della FMH, spiega a nome del CC, che questo non sostiene né le misure proposte né gli argomenti degli oppositori, anche se alcuni provengono da membri del CC. Nel corso della discussione emerge poco alla volta, e in modo sempre più chiaro, che si è pronti a considerare la possibilità di un aumento delle quote prima di prendere delle misure di risparmio dolorose o che danno dei segnali indesiderabili. Il successo riscontrato in occasione della manifestazione del 1 aprile 2006 è ricordato a più riprese, come anche l'élan di solidarietà che l'ha caratterizzato e reso possibile. Si costata anche che la FMH non "getta il denaro dalla finestra», considerato come un effetto secondario positivo dalle analisi effettuate. In questo spirito è fatta la proposta di mostrare chiaramente tutto quello che la FMH non sarebbe più in grado di fare se si riducesse il suo budget.

È presentata tutta una serie di proposte. 
Proposte n. 8/1-3 (P. Studer, ASMAC)

1. L'ASMAC Svizzera propone che si continui a rimborsare le quote dei membri senza cambiamento alcuno.

2. L'ASMAC Svizzera propone che le riserve costituite o disponibili nel settore Formazione, Perfezionamento professionale e Aggiornamento non siano utilizzate per dei compiti che non concernono la formazione, il perfezionamento professionale e l'aggiornamento.

3. L'ASMAC Svizzera propone che dopo l'applicazione delle misure che mirano ad accrescere l'efficienza della segreteria centrale, il deficit esistente sia coperto da un aumento delle quote dei membri, scaglionato a seconda della categoria di membro.

J. Nadig, Oncologia, propone che per coprire il deficit, invece del contributo di solidarietà (di un importo di 1,8 milioni di franchi), si utilizzi il contributo $P R$ restante (dell'importo di 1,1 milioni di franchi circa).

P. Wiedersheim, San Gallo, sostenuto da P. Meyrat, Soletta, chiede un sì chiaro per una $\mathrm{FMH}$ «forte», sotto forma di un chiaro aumento delle quote di membro scaglionato a seconda delle categorie di contributo.

Dopo una pausa J. de Haller precisa chiaramente che se la Camera dovesse decidere di applicare soltanto alcuni elementi delle misure proposte dal gruppo di lavoro, per esempio le misure di risparmio in seno alla SG, rifiuterebbe di piegarvisi per delle ragioni di ordine etico e sociale. Tanto più che è inaccettabile che si prendano delle misure dolorose da una parte, mentre dall'altra si dispone di milioni di franchi per delle «future eventualità». Secondo lui si deve ricorrere a questo denaro, quando è necessario e non tenerlo da parte quando è urgentemente necessario altrove. Siccome il dibattito è ben avanzato, il presidente fa una proposta sul modo di procedere.

Si vota sulla proposta n. 8/2 dell'ASMAC. J. de Haller precisa che si tratta soltanto dell'utilizzazione del contributo di solidarietà di un importo di 1,8 milioni di franchi e non dell'insieme delle riserve della FPA. La proposta è approvata con 102 voti favorevoli e 39 voti contrari, e testimonia la chiara volontà dei delegati di non toccare le risorse della FPA. È quindi messa ai voti la proposta $n$. 8/3 dell'ASMAC. Le misure di risparmio isolate sono respinte con 95 voti contrarie 49 favorevoli. Anche la proposta Nadig concernente l'utilizzazione del contributo destinato alle relazioni pubbliche è respinta con 75 voti contro 69, come anche la proposta $n$. 8/1 dell'ASMAC che chiede di non cambiare niente ai rimborsi, e questo con 93 voti contro 47. Questi risultati non permettono, così, di assicurare il finanziamento. J. de Haller propone di respingere l'insieme delle misure del pacchetto e di incaricare il gruppo di lavoro di elaborare un nuovo modello sulla base dei risultati delle votazioni appena effettuate. Questa proposta è accettata, con 143 voti favorevoli, 2 voti contrari e 1 astensione, con l'osservazione che il CC deve partecipare ai lavori.

Resta la proposta del gruppo di lavoro «Rimborso». Siccome rappresenta una parte dell'insieme delle misure respinte, non sarebbe logico approvarla. Su questa constatazione, la Camera medica respinge l'entrata in materia su questa proposta, con 2 voti favorevoli e 3 astensioni.

Si vota ora sulle proposte relative all'aumento delle quote di membro. Dai calcoli effettuati nel frattempo dalla SG risulta che sulla base delle statistiche dei membri al 1 gennaio 2006, per coprire il deficit di 1,5 milioni di franchi l'aumento della quota dovrebbe essere di Fr. 69.99, (ovvero Fr. 70.-). Sulla base di queste cifre e dello spazio a disposizione P. Wiedersheim propone un aumento una tantum della quota di membro per l'anno 2007, dell'importo di Fr. 100.-. Questa proposta è approvata con 117 voti favorevoli, 14 contrari e 5 astensioni.

Le quote dei membri già fissate sono quindi aumentate nel modo seguente per l'anno 2007: - categoria 1 e 2: aumento di $25+100=$ Fr. 125.-;

- categoria 3 e 4: aumento di $12+50=$ Fr. 62.-; - categoria 5 e 6: aumento di $6+25=$ Fr. 31.-

Per terminare si ringraziano ancora una volta $i$ gruppi di lavoro per il lavoro svolto. L'incarico affidato al gruppo di lavoro «Stabilizzazione» è completato in modo da:

- comprendere un chiarimento sull'utilizzazione delle riserve vincolate e di altre fonti di reddito (contributi, tasse, prezzi);

- fornire ai delegati alla Camera delle informazioni in una forma moderna e dettagliata;

- integrarsi nel processo budgetario del CC per il 2008.

La Camera medica conferma quindi all'unanimità con un'astensione che la direzione delle misure di stabilizzazione continua ad essere il compito del gruppo di lavoro.

Considerato il dibattito che si è appena tenuto, Felix Eymann precisa che auspica che le questioni di questo tipo siano trattate in modo più severo e rigoroso dall'Assemblea dei delegati. Per quel che concerne il seguito del programma, 


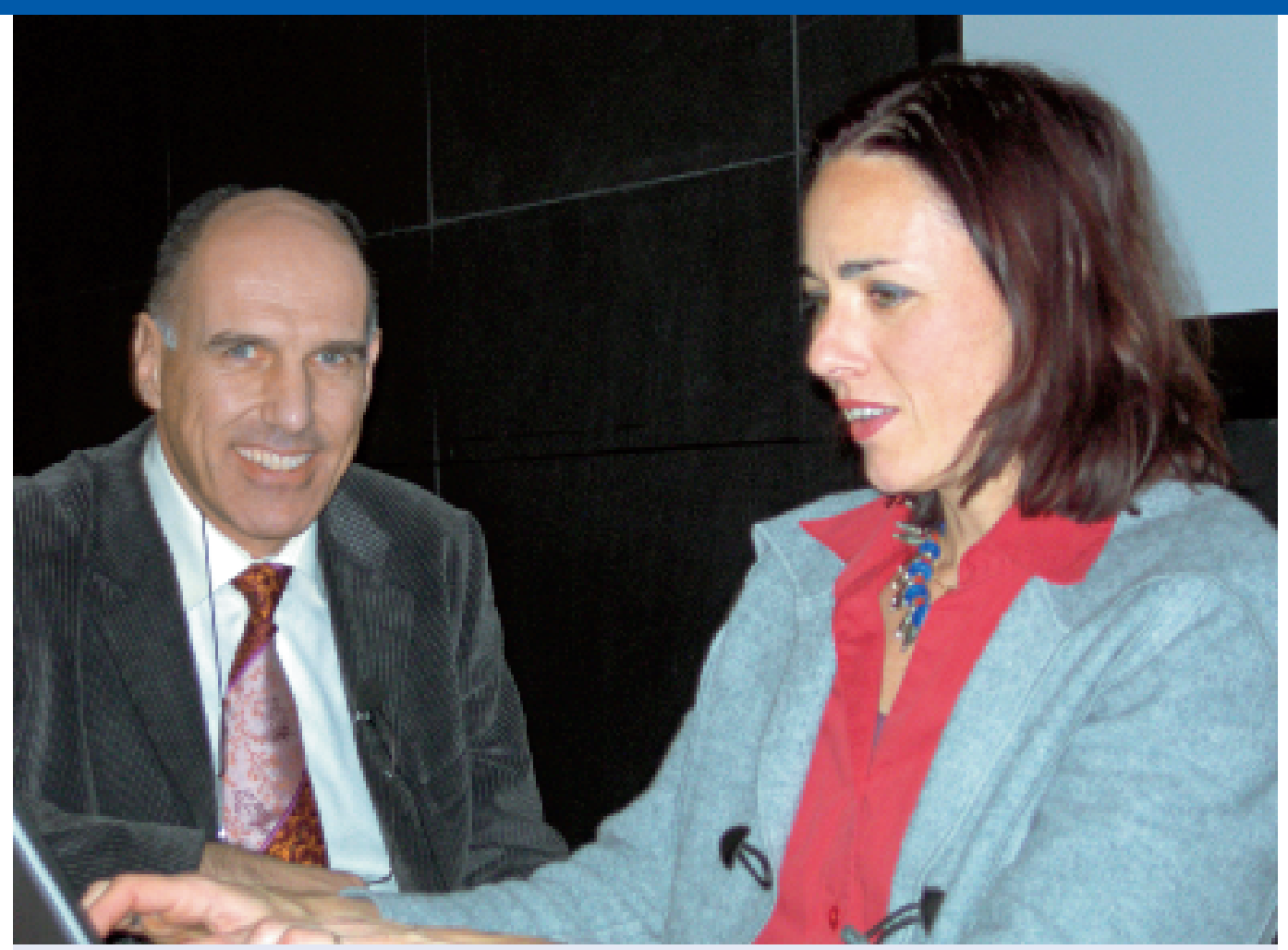

Jacques de Haller e Annamaria Müller Imboden, presidente e segretaria generale della FMH.

sono presentate diverse mozioni d'ordine, tra le quali la proposta n. 9.1/6 (H. Chaudhry, ASMAC), che chiede di trattare dapprima l'argomento del Managed Care e poi il regolamento di esecuzione. Se il tempo a disposizione non fosse sufficiente, si dovrebbe accettare provvisoriamente il regolamento di esecuzione così com'è sino al mese di maggio 2007. Per dei motivi tecnici, si potrà votare su questa proposta soltanto dopo aver trattato il punto seguente.

\subsection{Presentazione del progetto DINAMO}

Res Haefeli presenta il progetto posto sotto l'egida della Conferenza delle società cantonali di medicina (CSCM). Il progetto «DINAMO» trae il suo nome dalle nozioni di diagnosi, domanda («Nachfrage») e morbidità e deve permettere di mettere in luce, in modo sistematico e secondo diversi punti di vista, i fattori importanti rilevanti per fornire le prestazioni mediche. I «fattori» detti «di prestazione» permetteranno di fare una discussione differenziata sui costi e sulle quantità nel settore della salute - in particolare negli studi medici - nella speranza di abbandonare gli indicatori demografici insoddisfacenti come l'età e il sesso. R. Haefeli spiega brevemente i diversi aspetti sotto i quali si presentano i fattori di prestazioni.

- diagnosi: determinazione delle diagnosi tracer considerate importanti;

- domanda: avvio della prestazione, sistema marker;
- morbidità: TMI (ThurgauerMorbiditätsIndex Indice turgoviese di morbidità) per valutare lo stato di salute attuale e prospettivo di una persona.

Per finire, il relatore precisa che il suo progetto non è per niente qualcosa «nice to have», ma risponde ad una necessità urgente della nostra epoca. Questo progetto è portato avanti in stretta collaborazione con la FMH, in particolare con il servizio tariffario e il dipartimento DDQ.

È ora sottoposta a votazione la proposta $n$. 9.1/6 (Chaudhry), che è approvata con 72 voti favorevoli e 57 contrari.

\section{Politica sanitaria}

\subsection{Tesi concernenti il managed care}

Le tesi elaborate da un gruppo di lavoro della FMH che beneficiano di un vasto appoggio nel corpo medico, sono alla base di questo punto all'ordine del giorno. Queste tesi, che concernono le cure gestite (managed care) e l'obbligo di contrarre, sono state pubblicate nel Bollettino dei medici svizzeri e hanno sollevato una vasta eco. O. Kappeler, membro di questo gruppo di lavoro, presenta la genesi del documento e le tesi in esse contenute, le quali si basano sul carattere facoltativo e adattabile dei sistemi di gestione delle cure (managed care). L'utilità di questi sistemi è attestata, ma non è ancora stata provata scientificamente. Il dibattito sulla questione ha però 
avuto come conseguenza di far rinviare la questione sull'obbligo di contrarre alle Camere federali. Dopo l'approvazione da parte della Camera medica, nella prossima tappa si svilupperà ulteriormente il progetto, in particolare per quel che concerne il trasferimento del know-how necessario e destinato a evitare malintesi o interpretazioni erronee. O. Kappeler presenta quindi brevemente le proposte della Società bernese di medicina, della società svizzera di psichiatria e psicoterapia e della Società svizzera di ginecologia. Le esigenze che vi figurano sono accolte seriamente e se ne terrà debitamente conto, ma non presentano nessuna contraddizione seria con le tesi emesse. Per questo motivo il CC consiglia di respingerle. La presentazione di O. Kappeler è applaudita.

Le proposte sono presentate secondo l'ordine in cui sono arrivate:

\section{La proposta n. 9.1/2 (J. Schlup, Berna)}

approva il concetto «Managed Care - obbligo di contrarre», ma respinge espressamente l'introduzione di una responsabilità budgetaria, perché non è compatibile con l'etica medica.

La proposta n. 9.1/3 (H. Kurt, SSPP e FMPP) chiede un accesso diretto ai trattamenti psichiatrici e psicoterapeutici sia possibile anche nei modelli di managed care e nelle reti mediche, e che si presti un'attenzione particolare al trattamento delle persone colpite da una malattia psichica cronica in questi modelli e nelle reti (compensazione dei rischi). Questa richiesta è giustificata dalle specificità del trattamento psichiatrico-psicoterapeutico e di questo genere di pazienti.

La proposta n. 9.1/4, presentata da D. Stucki, Ginecologia e ostetricia

in assenza di Blaise Bourrit, chiede che nel sistema managed care i ginecologi/ostetrici siano non solo i medici di base per i controlli ginecologici e ostetrici e per le questioni relative alla contraccezione, ma anche per le malattie ginecologiche, Qualsiasi altra opzione sarebbe un'inutile perdita di tempo che non farebbe che aggravare la situazione.

\section{Per finire la proposta $n$. 9.1/5}

(J. Nadig, Oncologia)

chiede che il CC prenda delle misure per mantenere la qualità delle cure nelle reti che hanno una responsabilità budgetaria. Questi provvedimenti consisteranno nel misurare la qualità delle cure mediante mezzi appropriati nell'ambito di studi epidemiologici indipendenti. Si deve assicurare che le reti siano strutturate in modo che le decisioni terapeutiche concernenti i pazienti colpiti da tumori siano prese da una commissione interdisciplinare specializzata e che le raccomandazioni date dagli oncologi siano discusse con i pazienti.

O. Kappeler risponde alle argomentazioni fatte: - Una «responsabilità budgetaria» è contraria all'etica e bisogna quindi respingerla per questo motivo? La risposta è no, perché la coresponsabilità budgetaria (assunta volontariamente) è anche un settore nel quale è auspicabile acquisire esperienza nel senso preconizzato dalle tesi. Per esperienza si sa che ciò non avviene a scapito dell'etica e che un divieto non servirebbe a niente.

- Accesso diretto agli psicoterapeuti? Si prevede di mantenere uno accanto all'altro i modelli di managed care e i modelli convenzionali. Lo psicoterapeuta può anche intervenire in quanto gestore di cure, perché lo scopo delle cure integrate è ottimizzare la collaborazione.

- Accesso diretto ai ginecologi? Integrare degli specialisti significa che anche i ginecologi possono intervenire come gestori di cure. In alcune tappe della vita, il ginecologo è spesso il primo specialista a cui si rivolge una donna.

- Mantenere la qualità delle cure? Evidentemente questo deve restare il principio supremo. È, però, necessario intensificare la ricerca scientifica.

La discussione che segue non è molto accesa. $\mathrm{Ci}$ si domanda chi debba e possa agire come «gate keeper» e sono evocati i problemi di smistamento adeguato dei pazienti. Inoltre, alcuni criticano il fatto che l'aspetto dell'informazione appaia troppo poco nelle tesi presentate. L'integrazione degli specialisti prevista nelle tesi accoglie, invece, una grande adesione ed è accettato anche il fatto che l'eliminazione dell'obbligo di contrarre non sia più considerata una minaccia assoluta. Dei medici che praticano il managed care parlano delle loro esperienze e cercano di attenuare i timori dei loro colleghi per quel che concerne la corresponsabilità budgetaria. Il principale vantaggio di questi modelli non sta nei risparmi realizzati, ma nel miglioramento della collaborazione tra il medico di base e gli specialisti. Sarà tuttavia necessario un certo numero di anni per istaurare una relazione di fiducia che non può essere imposta di forza o nascere dal nulla.

J. Schlup è disposto a ritirare la proposta $n$. 9.1/2 della Società medica bernese se l'aspetto volontario e facoltativo è chiaramente espresso 
nelle tesi. Anche H. Kurt indica che la SSPP ritira la sua proposta $n .9 .1 / 3$, se viene assicurato che si terrà conto dei suoi interessi. La proposta della SGGO n. 9.1/4 viene votata, ma è respinta con 97 voti contrari, 18 favorevoli e 12 astensioni. J. Nadig è disposto a ritirare la seconda parte della proposta n. 9.1/5 degli oncologi, se la seconda parte, concernente la qualità delle cure, è accettata. Come è poi il caso, con 75 voti favorevoli e 26 contrari.

Per finire, la proposta n. 9.1/1 (Comitato centrale), concernente l'approvazione delle tesi «Managed Care - obbligo di contrarre» è messa in votazione ed è approvata, con riserva dei punti sopraindicati, con 112 voti favorevoli, 4 contrari e 7 astensioni.

\subsection{Partecipazione della FMH} all'Organizzazione mantello del mondo del lavoro per il settore sanitario (OdA Santé)

\section{La proposta n. 10/1 (M. Müller, CMB)}

chiede, a nome delle società mediche di base (CMB, SSMG, SSMI e SSPP) che la FMH deve fare in modo di poter far parte dell'OdA Santé (Organizzazione mantello del mondo del lavoro per il settore sanitario) e di collaborarvi attivamente. Quest'organizzazione è incaricata di definire e di configurare le professioni che, nel settore della sanità, dispongono già o disporranno di un diploma di scuola universitaria professionale o di scuola superiore. In un prossimo futuro, queste professioni potranno tangere o addirittura entrare in competizione diretta con diverse specializzazioni della medicina. M. Giger risponde che a proposito della Regolamentazione concernente la formazione delle assistenti medico (ex aiutomedico), la FMH ha già presentato all'OdA Santé la sua candidatura, ma senza successo. La FMH è un'organizzazione professionale ai sensi della LPMed, mentre l'OdA Santé disciplina soltanto le professioni poste sotto l'egida dell'UFFT. Se si parla di una categoria professionale specifica (come per esempio i soccorritori diplomati), è chiaro che la FMH intervenga nel dibattito, perché non è soltanto un'associazione professionale, ma anche un'associazione di datori di lavoro. M. Giger è del parere che sedere in permanenza all'OdA Santé non sia né opportuno né interessante per la FMH. In molti intervengono esprimendo un parere diverso. Far parte di quest'organizzazione significherebbe, infatti, informarsi attivamente. E per quanto l'OdA Santé esista anche sul piano cantonale e l'adesione delle società mediche cantonali sia molto raccomandata, non si deve trascurare il piano federale perché è lì che viene definita la struttura di base delle professioni.

Prima di votare su questa proposta, si deve decidere sull'entrata in materia, perché si tratta di un punto portato in un secondo tempo all'ordine del giorno. È presa la decisione di entrare in materia, con la necessaria maggioranza dei due terzi, con 113 voti favorevoli, 3 contrari e 5 astensioni. Si vota quindi su questa proposta che è approvata con 109 favorevoli, 2 contrari e 11 astensioni.

\section{Regolamento d'esecuzione della FMH}

In considerazione dell'ora (16.15), J. de Haller considera inutile lanciarsi in deliberazioni sul Regolamento d'esecuzione. Fa notare che la proposta n. 9.1/6 (Chaudhry, ASMAC) prevede l'applicazione provvisoria del nuovo Regolamento d'esecuzione sino alla prossima seduta ordinaria della Camera medica, e che i principali autori di proposte al punto 12 dell'ordine del giorno sono d'accordo di procedere in questo modo. T. Heuberger rincara che non si può «far fuori» in fretta e in qualche modo il Regolamento di esecuzione, ma che d'altra parte non si può più continuare a lavorare sulla base di una vecchia versione inutilizzabile del regolamento di esecuzione. Per la società medica bernese è quindi indispensabile che il Regolamento di esecuzione sia discusso in modo dettagliato in maggio. Idealmente si potrebbe integrarvi le esperienze fatte. J. de Haller chiede se il progetto di Regolamento d'esecuzione sottoposto alla Camera possa essere utilizzato a titolo provvisorio e interinale sino alla prossima seduta della Camera del 3 maggio 2009. Questa proposta è approvata all'unanimità con 109 voti favorevoli e 1 astensione.

\section{Varie}

J. de Haller informa che A. Müller Imboden, segretaria generale della FMH, ha dato le sue dimissioni per la fine dell'anno e che con un termine di licenziamento di sei mesi, lascerà le sue funzioni a metà del 2007. La ringrazia per il suo impegno e i delegati per la loro pazienza e chiude la seduta alle 16.30 . 